\title{
Injectable Botulinum Toxin as a Treatment for Plantar Hyperhidrosis
}

\section{A Case Study}

\section{Tracey C. Vlahovic, DPM* \\ S. Patrick Dunn, BS* Jill C. Blau, DPM† Caroline Gauthier, DPM¥}

\begin{abstract}
Hyperhidrosis is defined as excessive and uncontrollable sweating due to overactivity of the eccrine sweat glands. The first line of treatment for plantar hyperhidrosis consists of conservative therapies such as topical solutions (ie, antiperspirant applications and aluminum chloride preparations) and iontophoresis. When the patient has failed these standard treatments, the other available medical options are rather limited and not well tolerated. Botulinum toxin type A (Botox, Allergan Inc, Irvine, California) is a purified neurotoxin complex approved by the US Food and Drug Administration in 2004 for multiple medical conditions, including severe primary axillary hyperhidrosis that failed conservative topical therapies. Few recent clinical studies have suggested that botulinum toxin is effective in the treatment of plantar hyperhidrosis. In this case study, two patients received intradermal injections of botulinum toxin type $A$ into the plantar aspect of both feet. A 3-month follow-up evaluated the efficacy of botulinum toxin type A by subjectively assessing the amount of residual sweating. In these two patients, botulinum toxin type A was an effective and safe treatment for plantar hyperhidrosis. (J Am Podiatr Med Assoc 98(2): 156-159, 2008)
\end{abstract}

Plantar hyperhidrosis is a common medical and cosmetic concern. It can cause quality-of-life issues that may lead to social embarrassments. But more importantly, it may result in skin maceration and fungal and bacterial foot infections. Hyperhidrosis in the lower extremity has been reported to occur in $44 \%$ of all patients seeking medical treatment for hyperhidrosis; only axillary and palmar hyperhidrosis are more common. ${ }^{1}$ Standard conservative therapies for plantar hyperhidrosis include topical solutions, antiperspirant applications, aluminum chloride preparations, and iontophoresis. ${ }^{1,2,3}$ Because of their significant side effects and risks, other treatment options such as systemic anticholinergic agents and betablockers, as well as surgical sympathectomy are not often used..$^{4,5}$

Recently, researchers have demonstrated that bot-

*Temple University School of Podiatric Medicine, Philadelphia, PA.

†Virtua Health Systems, Camden, NJ.

\$Cambridge Health Alliance, Cambridge, MA.

Corresponding author: Tracey C. Vlahovic, DPM, Temple University School of Podiatric Medicine, 8th and Race Streets, Philadelphia, PA 19107. ulinum toxin type A (BTX-A) can be used successfully in the treatment of plantar hyperhidrosis. ${ }^{6}$ Botulinum toxin type $\mathrm{A}$ is a protein that acts by blocking the release of presynaptic acetylcholine at the neuromuscular junction. Inhibition of acetylcholine release blocks the excessive sympathetic stimulation of eccrine glands, which results in reduction of sweating in patients with hyperhidrosis..$^{3,7}$

\section{Materials and Methods}

The noncosmetic version of BTX-A (Botox, Allergan Inc), 100 units/vial, was used. The neurotoxin arrives in a vacuum-dried form that must be reconstituted with nonpreserved sterile saline (Fig. 1).

\section{Reconstitution of Botulinum Toxin}

An 18-gauge needle connected to a 5-mL syringe is used to inject $4 \mathrm{~mL}$ of nonpreserved sterile $0.9 \%$ sodium chloride at a $45^{\circ}$ angle into the BTX-A vial (Fig. 2). Using this amount of saline yields 2.5 units of BTX-A in $0.1 \mathrm{~mL}$ of the reconstituted solution. The syringe is then removed, allowing the air pressure to be- 


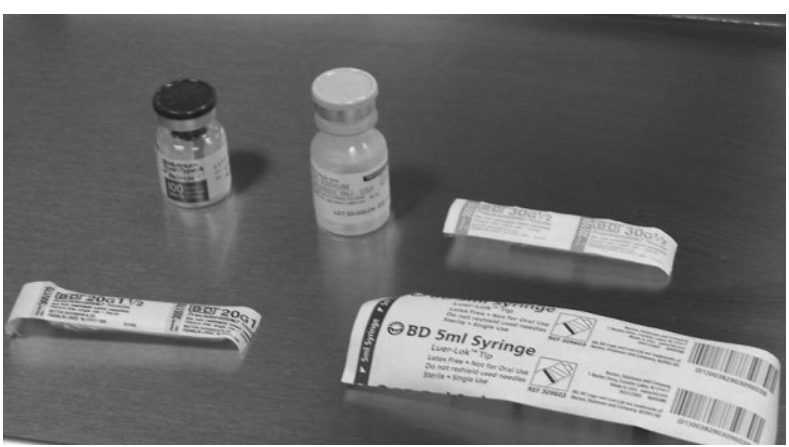

Figure 1. The nonreconstituted botulinum toxin type $A$ vial and nonpreserved saline vial.

come normative with the needle still intact. The vial is then gently swirled, never inverted, in order to mix the contents. Once completed, the 18-gauge needle is replaced with a 30-gauge needle and the mixture is ready for injection (Fig. 3). Once reconstitution has taken place, the solution should be used within 4 hours and should be stored in a refrigerator until use.

\section{Minor's lodine Starch Test}

The Minor's iodine starch test is used to determine the focal areas of hyperhidrosis. Iodine solution is evenly painted onto the plantar feet. Corn starch is dusted onto the painted areas with a simple cosmetic brush. The areas of greatest hyperhidrosis turn dark purple to black in color (Figs. 4 and 5).

\section{Injection Technique}

We recommend doing an ankle block prior to injection of BTX-A. Following this, topical lidocaine jelly with plastic wrap used for occlusion is applied for ap-

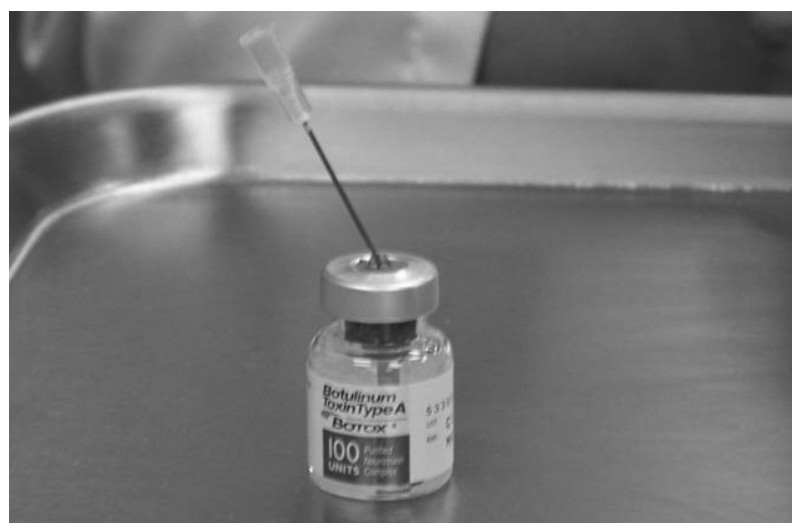

Figure 3. Following reconstitution, the syringe is removed and air pressure is allowed to equalize in the botulinum toxin type A solution.

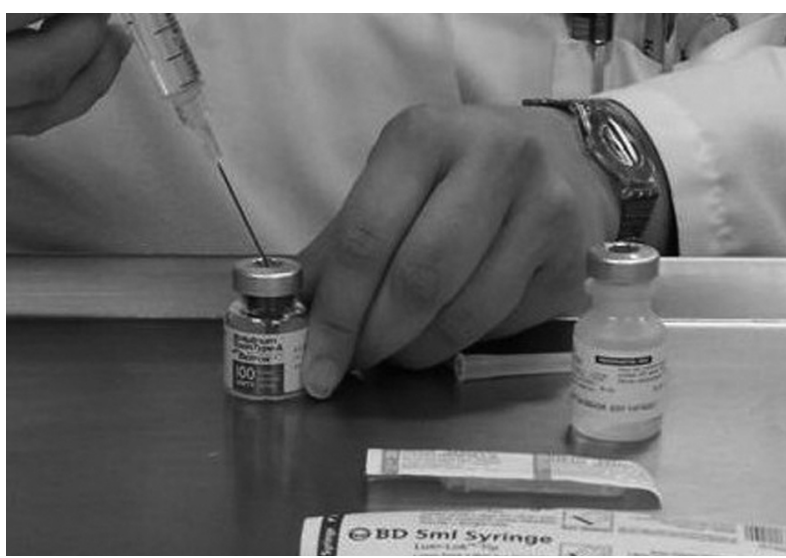

Figure 2. Reconstitution of botulinum toxin type $A$ (BTX-A) by injecting $4 \mathrm{~mL}$ of saline into BTX-A vial.

proximately 30 minutes in order to minimize the pain involved during the injection process. The Minor's iodine starch test is performed prior to injection. Following the test, the foot is cleaned with isopropyl alcohol and a grid (using a surgical marking pen) of dots $2 \mathrm{~cm}$ apart are marked over the areas of greatest hyperhidrosis (Fig. 6). The BTX-A reconstituted solution is drawn up by not inverting the bottle in 1-mL syringes (Fig. 7). Botulinum toxin type $\mathrm{A}$ is then injected intradermally at a depth of $3 \mathrm{~mm}$ with special care not to inject into the pen dot in order to avoid permanent tattooing (Fig. 8). Care should also be taken to avoid the plantar musculature in order to avoid muscular paralysis. Also, it is imperative to inject both the plantar aspect of the digits as well as in between the digits if hyperhidrosis is present in those areas. Approximately 35 units of BTX-A are commonly used on each foot. The pen marks are then cleaned from the foot and the procedure is finished. The first follow-up is scheduled 1 month after the procedure to check the level of the patient's improvement, and the second post-injection visit is scheduled 3 months later to check for any rebound hyperhidrosis. At the 3month visit, patients were asked to grade their improvement using the conditions outlined in Table 1. Generally, the second BTX-A treatment occurs 6 months after the initial treatment.

\section{Case Report 1}

A 35-year-old female presented to the Foot and Ankle Institute, Temple University School of Podiatric Medicine, Philadelphia, Pennsylvania for care of excessive plantar sweating for several years. Her mother had the same condition and had received BTX-A treatment. The patient did not complain of excessive 


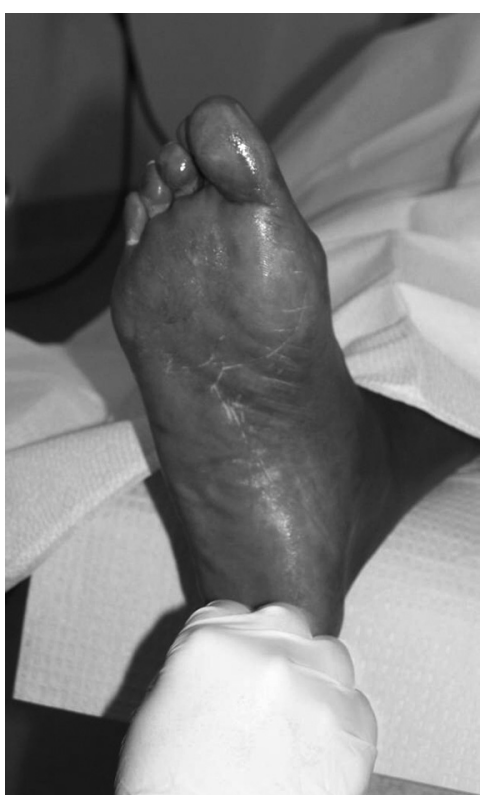

Figure 4. lodine is applied in an even layer to the plantar aspect of the foot.

sweating anywhere else on the body. The patient had failed topical aluminum chloride therapy, antiperspirants, topical antifungal medications, and iontophoresis. The patient desired BTX-A therapy to treat her hyperhidrosis. The patient received a total of 75 units of the toxin into the plantar aspect of both feet. The patient tolerated the procedure well with no residual hematoma or paralysis. Follow-up 3 months later re-

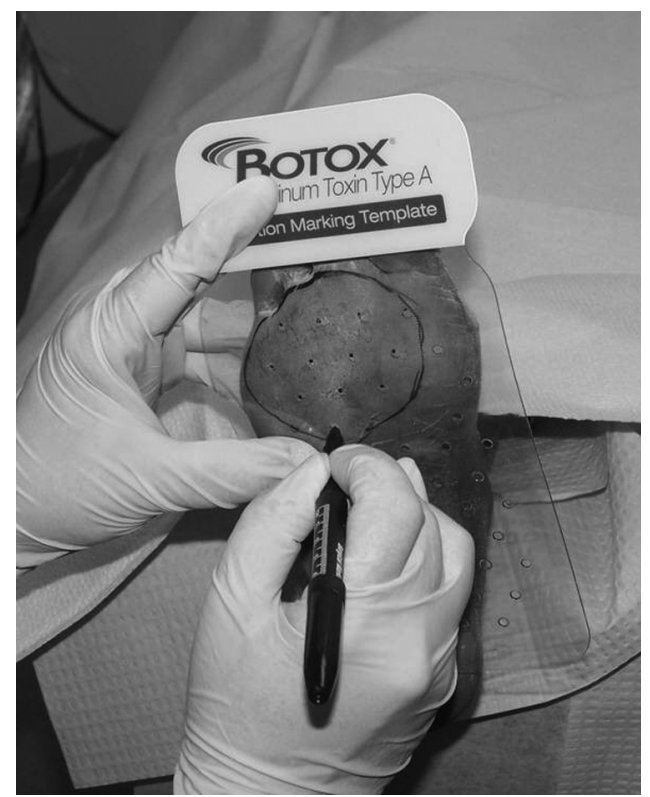

Figure 6. Once the areas are indentified, a surgical marking pen is used to mark dots spaced $2 \mathrm{~cm}$ apart.

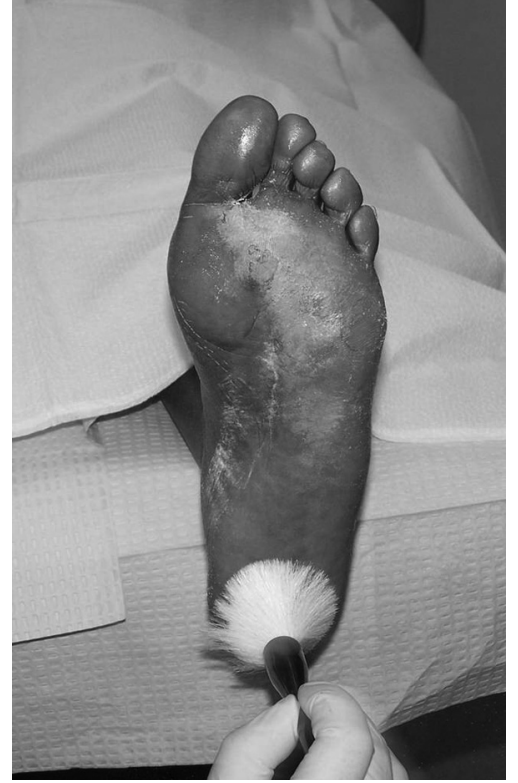

Figure 5. Corn starch is then dusted on top of the iodine solution to mark the areas of focal hyperhidrosis.

vealed a minor increase in sweating from initial treatment. Her satisfaction was a +3 or substantial improvement over the baseline (Table 1). ${ }^{8}$ At 6 months, the patient was satisfied with the injection-induced decrease in sweating but decided to start using aluminum chloride therapy instead of undergoing injection therapy again.

\section{Case Report 2}

A 49-year-old male presented to the Foot and Ankle Institute, Temple University of Podiatric Medicine,

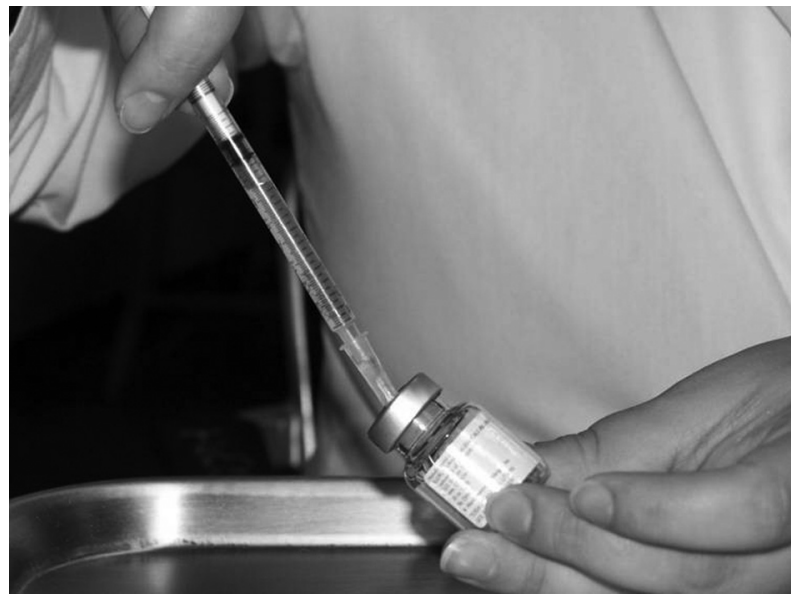

Figure 7. The reconstituted botulinum toxin type A solution is drawn up; it should be noted that the bottle should not be inverted during this process. 


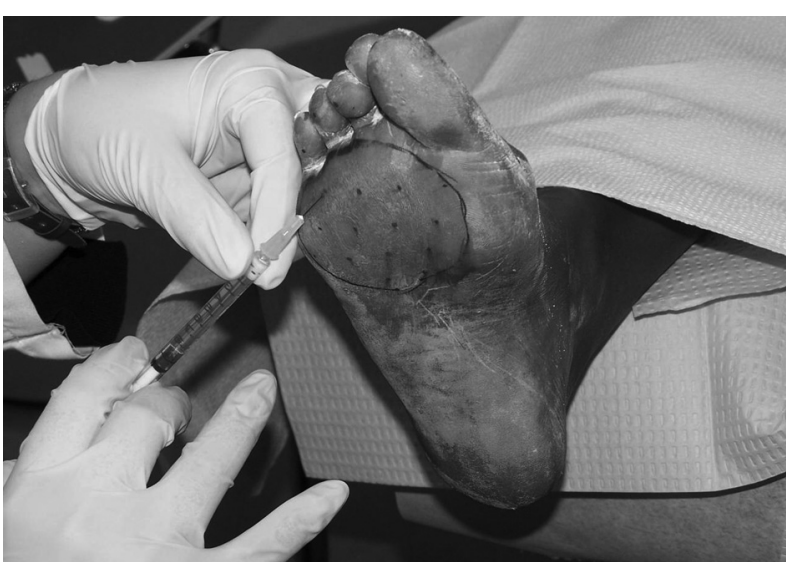

Figure 8. Approximately $0.1 \mathrm{~mL}$ is injected into the dermis, with a 30 -gauge needle, slightly below each dot.

Philadelphia, Pennsylvania, with a 6-year history of plantar hyperhidrosis. His medical history was negative for diabetes and hyperthyroidism. He complained of hot and sweaty feet on a daily basis regardless of season or shoe gear. Past treatment included overthe-counter topical therapy, antiperspirant, deodorant, aluminum chloride, topical clindamycin solution, and topical antifungal medication. While the patient was in the examination room, the patient's feet had continuous visible sweat droplet production. The patient desired BTX-A therapy to decrease his sweating. Minor's iodine starch test revealed concentrated areas of hyperhidrosis at the forefoot and mid-arch, but the heel area was spared. A total of 70 units were injected into both feet. Follow-up 3 months later revealed minor residual sweating. The patient's satisfaction rating was +3 (Table 1). The patient presented for a second BTX-A therapy 6 months after initial treatment due to recurrence of hyperhidrosis, albeit at a slightly decreased level than baseline.

\section{Discussion}

Both cases suggest BTX-A is an effective and safe treatment for hyperhidrosis that has failed topical therapy. Both patients related decreased sweating that did not regress to the baseline until close to 6 months after injection. In order to continue their remission, patients do need to continue the injection therapy. Using ice for local anesthesia as opposed to an ankle/ wrist block when performing bilateral botox injections has been advocated. ${ }^{9}$ However, other authors claim that even with cryoanesthesia, topical anesthesia, or both, the injection pain is intolerable. ${ }^{7}$ Because of this possibility, we chose to use regional anesthesia coupled with topical anesthesia prior to injecting botox.
Table 1. A modification of Naumann and Lowe's ${ }^{8}$ patient assessment of treatment satisfaction scale

\begin{tabular}{rl}
\hline Score & \multicolumn{1}{c}{ Description } \\
\hline+4 & $100 \%$ improvement, no signs or symptoms \\
+3 & $75 \%$ improvement, very few symptoms \\
+2 & $50 \%$ improvement, moderate signs and symptoms \\
+1 & $25 \%$ improvement, minimal signs and symptoms \\
0 & No change \\
-1 & $25 \%$ worse \\
-2 & $50 \%$ worse \\
-3 & $75 \%$ worse \\
-4 & $100 \%$ worse \\
\hline
\end{tabular}

Although BTX-A is expensive ( $\$ 485$ for a 100 -unit vial), it was covered by insurance for hyperhidrosis recalcitrant to topical therapy in the two patients presented. Due to variations in insurance coverage and a short shelf life, it is encouraged that patient eligibility for BTX-A treatment be verified prior to initiating therapy. Overall, BTX-A treatment is an appropriate treatment for patients with recalcitrant plantar hyperhidrosis.

Financial Disclosures: None reported.

Conflict of Interest: None reported.

\section{References}

1. Lear W, Kessler E, Solish N, et al: An epidemiological study of hyperhidrosis. Dermatol Surg 33: S69, 2007.

2. Lowe NJ, YAMaUchi PS, LASK GP, ET AL: Efficacy and safety of botulinum toxin type a in the treatment of palmar hyperhidrosis: a double blind, randomized, placebocontrolled study. Dermatol Surg 28: 822, 2002.

3. VADOUD-SEYEdI, J: Treatment of plantar hyperhidrosis with botulinum toxin type A. Int J Dermatol 43: 969, 2004.

4. LAI YT, Yang LH, Chio CC, et aL: Complications in patients with palmar hyperhidrosis with transthoracic endoscopic sympathectomy. Neurosurgery 41: 110, 1997.

5. SaAdia D, Voustianiouk A, Wang AK, et al: Botulinum toxin type A in primary palmar hyperhidrosis. Neurology 57: 2095, 2001.

6. Sevim S, Dogu O, Kaleagasi H: Botulinum toxin-A therapy for palmar and plantar hyperhidrosis. Acta Neurol Belg 102: 167, 2002.

7. Perez-Bernal A, Avalos-Peralta P, Moreno-Ramirez D, ET AL: Treatment of palmar hyperhidrosis with botulinum toxin type A : 44 months of experience. J Cosm Derm 4: 163, 2005.

8. Naumann M, Lowe NJ: Botulinum toxin type A in treatment of bilateral primary axillary hyperhidrosis: randomised, parallel group, double blind, placebo controlled trial. Br Med J 323: 596, 2001.

9. Sмith KC, Comite SL, AND Storwick GL: Ice minimizes discomfort associated with injection of botulinum toxin type A for the treatment of palmar and plantar hyperhidrosis. Dermatol Surg 33: S88, 2007. 